Case Report:

\title{
Huge Intra-abdominal Meningocele in a Patient With Neurofibromatosis 1 With Previous Posterior Spinal Fusion and Instrumentation Due to Severe Scoliosis
}

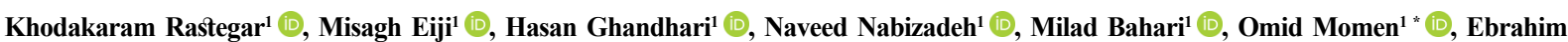
Ameri $^{1}$

1. Bone and Joint Reconstruction Research Center, Shafa Orthopedic Hospital, Iran University of Medical Science, Tehran, Iran.

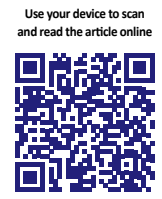

Citation Rastegar Kh, Eiji M, Ghandhari H, Nabizadeh N, Bahari M, Momen O, et al. Huge Intra-abdominal Meningocele in a Patient With Neurofibromatosis 1 With Previous Posterior Spinal Fusion and Instrumentation Due to Severe Scoliosis: A Case Report. Journal of Research in Orthopedic Science. 2019; 6(4):31-34. http://dx.doi.org/10.32598/JROSJ.6.4.73

: http://dx.doi.org/10.32598/JROSJ.6.4.73

\section{(c) (1) (\$)}

Article info:

Received: 03 Apr 2019

Revised: 23 Apr 2019

Accepted: 14 Sep 2019

Available Online: 01 Nov 2019

Keywords:

Meningocele,

Neurofibromatosis 1

Scoliosis

\begin{abstract}
A B S T R A C T
Introduction: Anterior Lumbar Meningoceles (MCs) are rare in the patient with Neurofibromatosis type 1 (NF1). Although spinal fusion with optimal resection of the meningocele might be indicated in these special cases, no report could completely describe a huge meningocele after posterior spinal instrumented fusion.

Case Presentation: Here we present a 23 years old woman with a history of NF-1 and previous posterior spinal fusion, who was referred to the neurosurgery department because of abdominal pain and retroperitoneal mass secondary to large anterior lumbar MC. The MC was filling the retroperitoneal cavity and protruded to the anterior wall of abdomen. As an elective procedure, reduction and closure of cyst was achieved through posterior approach. Postoperatively, the patient reported satisfactory relief of abdominal pain.
\end{abstract}

Conclusion: Lumbar anterior MC is rare in the patients with NF1. When surgical intervention is indicated, reduction of cele should be considered.

\section{Introduction}

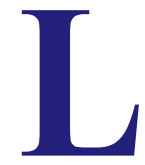

arge anterior lumbar Meningoceles (MC) are rare lesion associated with connective tissue disorders. Neurofibromatosis is a hereditary multisystem disease that may manifest itself as abnormalities of the skin, nervous tissue, bones, and soft tissues [1]. Neurofibroma- tosis causes formation of nerve tissue tumors, developing anywhere in the nervous system, including brain, spinal cord and peripheral nerves [2].

The term spinal meningocele are generally used to describe a congenital spinal malformation including protrusion of the spinal meninges from the congenitally dystrophic vertebra. The most common connective tissue, those

* Corresponding Author:

Omid Momen, PhD.

Address: Bone and Joint Reconstruction Research Center, Shafa Orthopedic Hospital, Iran University of Medical Science, Tehran, Iran.

Phone: +98 (911) 1777740

E-mail:dromid.momen@yahoo.com 


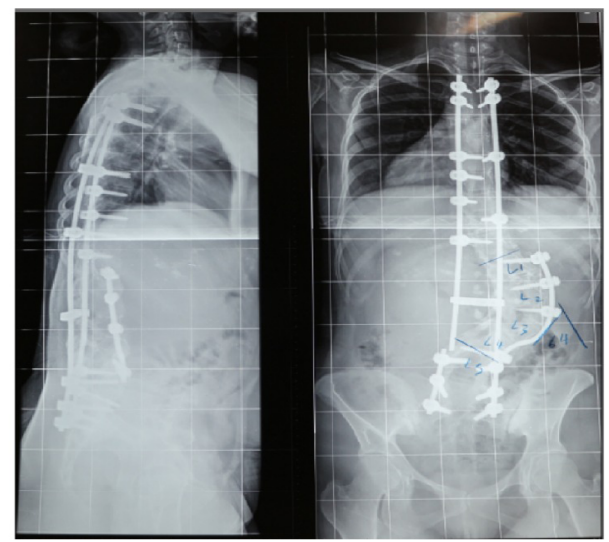

Orthopedic Science

Figure 1. The long cassette $x$ - radiograph

are usually associated with MC consist of neurofibromatosis (von Recklinghausen' disease) and Marfan syndrome [1, 2]. Neurofibromatosis is usually diagnosed in childhood or early adulthood. Neurofibromatosis-1 (NF-1), previously named as von Recklinghausen's disease, is an autosomal dominant disorder with an incidence of 1 in 3,500 births, equally prevalent among men and women [3].

Although it manifests with variable signs and symptoms, however, mainly affects the skin and peripheral nervous system leading to the neural tumor growth $[4,5]$. Some unusual tumors that occur with higher frequency in NF-1 include carcinoid, pheochromocytoma, brain tumors, and malignant peripheral nerve sheath tumors. Furthermore, other common tumors, such as lung, breast, colon, and prostate, are less frequent [6].

The tumors are usually noncancerous (benign), but sometimes can become cancerous (malignant); symptoms are often mild. Since this disorder is associated with neural tumor predisposition, the detection of a mass-like lesion increases the awareness regarding potentially malignancy, whenever

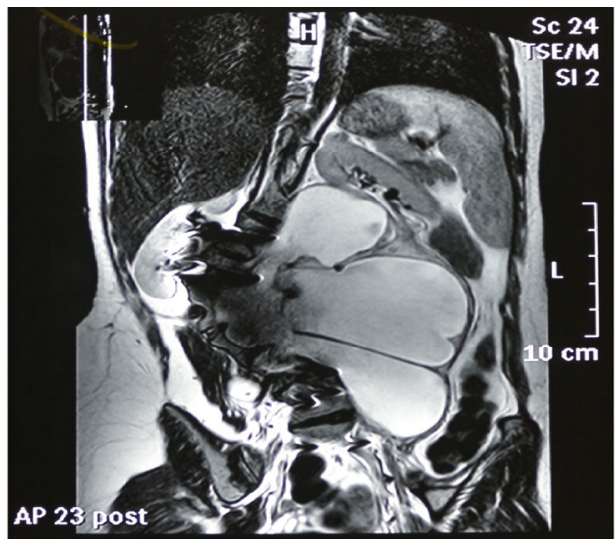

Figure 3. MRI (Coronal view)

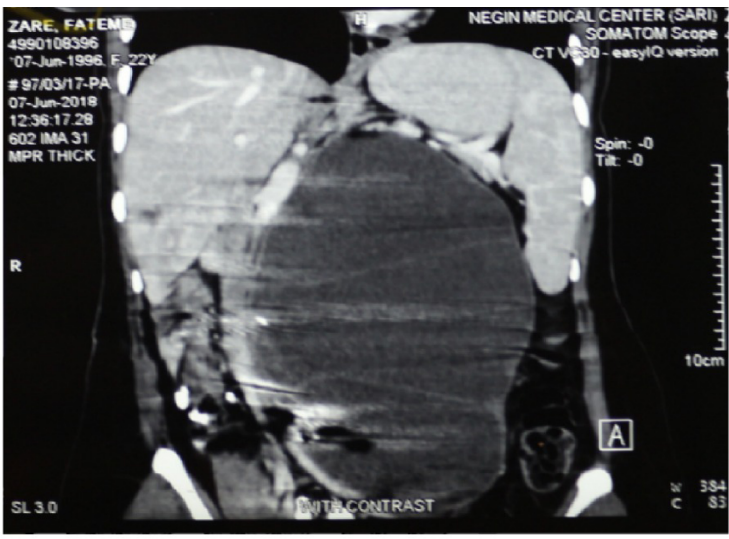

Figure 2. CT Scan (Coronal view)

Orthopedic Science

neurofibriomatosis tumor causes neural function impairment or space occupying lesion in circumstances that surgical intervention is implemented. In this report, we describe a case of NF-1 presenting with a large retroperitoneal mass that led to abdominal discomfort.

\section{Case Presentation}

A 23-years old woman, a known case of neurofibromatosis type 1 (NF1), referred with abdominal fullness and severe pain. She reported a history of instrumented spinal fusion due to kyphoscoliosis from 10 years ago. A physical examination showed she had widespread cafe-au-lait spots, cutaneous neurofibroma, axillary freckles, and lisch nodules in her iris. A long cassette x-radiographs demonstrated instrumented fusion mass with somehow residual deformity and soft tissue mass (Figure 1).

The CT scan (Figure 2) and MRI (Figure 3) showed a 30 $\mathrm{cm}$ homogenous cystic mass with a thin wall extended from T12, L1 dura into the retroperitoneal space through the foraminal corridor forward abdominal exam. She underwent meningocele volume-diminishing and procedure-diminishing meningocele surgery with only posterior approach without any unwanted incident. The surgical approach was accomplished through foraminal access and proceeding to the latteral extra-cavitary space toward the retroperitoneal fossa. Excision of the protruded cyst wall and its contents was performed to reduce the size of the cele.

Postoperatively, her abdominal fullness and pain was dramatically relieved and the cyst was remained small in follow up examination. 


\section{Discussion}

A spinal meningocele is an extended protrusion of the meninges through a dilated intervertebral foramen or a bony defect of the vertebral column. Although it is relatively common after tearing of dura during laminectomy laminectomy, however, congenital meningocele is relatively rare and usually associated with generalized mesenchymal dysplasia, such as NF-1 [7]. Thoracic and/or lumbar spinal levels are the most common localization. Because of given their insidious nature, $\mathrm{MC}$ can be presented as a quite large mass. Anterolateral cervical localization is rare.

Most patients with meningocele are asymptomatic, but clinical manifestations can develop depending on the size and location. Intra-abdominal meningoceles are not true abdominal tumors or cysts; they are diverticular of the spinal meninges that protrude through the neuroforamen. It may compress the lung and mediastinal structures, abdominal structure. Cervical structures can cause cough, dyspnea, palpitation, abdominal pain, and stridor [6].

Meningocele repair (also known as myelomeningocele repair) details the repair of birth defects of the spinal cord and spinal membranes. Meningocele and myelomeningocele are types of spina bifida. Operation is indicated if the lesion causes symptoms due to compression of surrounding structure by the meningocele or when the size of MC is rapidly increasing. Detailed radiological assessment of meningocele is necessary since it may associate with neuroma in the sac. Magnetic resonance appearances of meningocele is diagnostic and differentiate it from posterior retroperitoneal tumor and intra-abdominal tumor such as neurofibroma, neuroblastoma, and ganglioneuroma predisposes NF-1 predisposes a high risk of tumor formation.

Although laminectomy and intra dural repair of cyst is sufficient for small lesions, transthoracic approach for large ones is used to reduce complication such as cord damage or meningocele fistula.

In conclusion, intra-abdominal meningocele is rare and benign pathology. Surgical treatment is sometimes needed for symptomatic growing lesions. Meningocele should be considered in NF-1 patients presenting with mass-like lesion.

\section{Funding}

This research did not receive any specific grant from funding agencies in the public, commercial, or not-forprofit sectors.

\section{Authors' contributions}

All authors contributed in preparing this article.

\section{Conflict of interest}

The authors declared no conflict of interest.

\section{References}

[1] Dadlani R, Sadanand V, Ghosal N, Hegde AS. Congenital giant plexiform neurofibroma with occipital calvarial dysplasia in association with meningoencephalocele in neurofibromatosis type 1 and segmental neurofibromatosis: Report of 2 cases. J Neurosurg Pediatr. 2013; 12:458-64. [DOI:10.3171/2013.8.PEDS12624] [PMID]

[2] Jett K, Friedman JM. Clinical and genetic aspects of neurofibromatosis 1. Genet Med. 2010; 12:1-11. [DOI:10.1097/ GIM.0b013e3181bf15e3] [PMID]

[3] Tonsgard JH. Clinical manifestations and management of neurofibromatosis type 1. Semin Pediatr Neurol. 2006; 13:2-7. [DOI:10.1016/j.spen.2006.01.005] [PMID]

[4] Wallace MR, Marchuk DA, Andersen LB, et al. Type 1 neurofibromatosis gene: Identification of a large transcript disrupted in three NF1 patients. Science. 1990; 249:181-86. [DOI:10.1126/ science.2134734] [PMID]

[5] Ferner RE. Neurofibromatosis 1 and neurofibromatosis 2: A twenty first century perspective. Lancet Neurol. 2007; 6:340-51. [DOI:10.1016/S1474-4422(07)70075-3]

[6] Sorensen SA, Mulvihill JJ, Nielsen A. Long-term follow-up of von Recklinghausen neurofibromatosis: survival and malignant neoplasms. N Engl J Med. 1986; 314:1010-5. [DOI:10.1056/ NEJM198604173141603] [PMID]

[7] Oner AY, Uzun M, Tokgoz N, Tali ET. Isolated true anterior thoracic meningocele. AJNR Am J Neuroradiol. 2004; 25:1828-30.

\section{Ethical Considerations}

\section{Compliance with ethical guidelines}

All ethical principles were considered in this article. 
This Page Intentionally Left Blank 